\title{
Conteneurisation de matériaux de dragage dans des géocontainers (B) en vue de leur immersion.
}

\author{
Olivier Briand \\ Docteur-ès-sciences, Société Géomer, Marseille \\ Denis Gieulles \\ Directeur, Société Géomer, Marseille
}

\section{Résumé :}

La conteneurisation de matériaux dans des structures en géotextiles est un concept qui intéresse de plus en plus les gestionnaires de ports et les administrations concernées par l'évacuation des déblais de dragage. L'immersion de ces déblais de dragage est strictement réglementée et l'autorisation d'immersion au large, de plus en plus rare, surtout lorsque ces matériaux contiennent de fortes concentrations en métaux lourds. La solution de confinement des matériaux pollués, réduit les risques liés à la mobilité des particules du sol immergé. L'utilisation de nappes de confinement a été expérimentée avec succès lors des travaux de dragage du port des Marines de Cogolin en 1995 (Var, France).

\section{Introduction}

Le concept de conteneurisation de matériaux intéresse de plus en plus les gestionnaires de ports et les administrations concernées par l'évacuation des déblais de dragage. L'immersion de ces déblais de dragage est strictement réglementée et l'autorisation d'immersion au large, de plus en plus rare, surtout lorsque ces matériaux contiennent de fortes concentrations en métaux lourds. En effet, les risques de retour à la côte par mise en suspension de fines au cours du largage ou de contamination des fonds environnants sous l'action des courants, sont importants. Pour pallier à ces inconvénients, la solution de confinement des matériaux pollués, réduit les risques liés à la mobilité des matériaux.

Le cas que nous exposons concerne l'opération de dragage et de rejet par immersion des matériaux du port des Marines de Cogolin dans le Var. Les matériaux proviennent du petit fleuve Le Bourrian qui se jette dans le port. Par l'ensablement des bassins qu'ils provoquent, ces matériaux génèrent une réduction importante du plan d'eau utilisable puisque le tirant d'eau est par endroit, inférieur à $0.8 \mathrm{~m}$. Le dragage de $5000 \mathrm{~m} 3$ de matériaux était prévu. L'autorisation d'immersion a été donnée par la préfecture après avis des services de l'état compétents en la matière. Le point d'immersion autorisé se situait au large de la baie de St Tropez par des fonds de $-180 \mathrm{~m}$. 
Cette technique de confinement consiste à stocker les matériaux contaminés dans de grands conteneurs souples appelés Géocontainers $\mathbb{B}$. Ces enveloppes, de $30 \mathrm{~m}$ de long et de $6 \mathrm{~m}$ de diamètre, sont réalisées en géotextile tissé dont la résistance mécanique est importante et la porométrie adaptée à la granulométrie des sédiments. Une fois le Géocontainer (B) rempli de $350 \mathrm{~m} 3$ de sédiments, le chaland achemine son chargement vers la zone de largage. Pendant le trajet, une équipe referme le conteneur par des coutures triples rangs en ménageant un soufflet replié vers lintérieur du sac dont les coutures moins résistantes en se rompant absorberont une partie du choc sur le fond. Malheureusement, la procédure d'immersion a été interrompue par le préfet sous la pression des marins pêcheurs professionnels après limmersion du premier Géocontainer (B. Cette expérimentation n'ayant pas été achevée, nous n'avons pas pu en retirer toutes les connaissances et l'expérience que nous avions envisagées. Deux autres Géocontainers $\mathbb{Q}$ ont été utilisés et clapés dans l'enceinte du port .

\section{Matériaux uêilisés}

\subsection{Le Géocontainer (1)}

\subsubsection{Le textile}

Le tissu employé pour la confection du sac, produit NICOLON B.V., est un géotextile tissé dont la matière première est du polyéthylène haute densité pour la chaîne et du polyester pour la trame. Le tableau I indique les caractéristiques du géotextile

\begin{tabular}{|c|c|c|}
\hline & & Nornes \\
\hline Matière première & Chaîne : Polyéthylène & \\
\hline \multirow[t]{2}{*}{ Résistance à la traction $(\mathrm{kN} / \mathrm{m})$} & Chaine : 90 & \\
\hline & Trame : 90 & DN 53857 \\
\hline \multirow[t]{2}{*}{ Allongement à la rupture $(\%)$} & Chaine : 25 & \\
\hline & Trame : 20 & \\
\hline Permittivité $(\mathrm{L} / \mathrm{m} 2 / \mathrm{s})$ & 10 & NEN 5167 \\
\hline Porométrie $(\mu \mathrm{m})$ & 100 & NEN 5168 \\
\hline Masse surfacique $(\mathrm{g} / \mathrm{m} 2)$ & 400 & DIN 53854 \\
\hline Epaisseur (mm) & 1,2 & DNN 53855 \\
\hline
\end{tabular}

Tableau I caractéristiques du géotextile d'après Prodireg Sarl 


\subsubsection{Porométrie et rétention des particules}

La condition de filtration doit répondre aux critères de non-entrainement des particules du sol. Pour valider le choix du géotextile, on compare l'ouverture de filtration Of du géotextile à la dimension des plus grosses particules susceptibles de la traverser, dimension déterminée par le d 85 du sol à filtrer.

$$
\text { soit } \text { of }<\mathrm{C} * \mathrm{~d} 85
$$

Avec: d $85=$ diamètre des éléments du sol à filtrer tel que $85 \%$ des éléments de ce sol soient inférieurs à ce diamètre.

$\mathrm{C}=$ coefficient adimensionnel tenant compte de la granulométrie, de la densité du sol, du type d'écoulement hydraulique et du rôle du géotextile. [1]

Les caractéristiques granulométriques et physico-chimiques du sol (Tableau I, III et Figure 1) donnent pour le d 85 une valeur de $8 \mathrm{~mm}$. La valeur de $C$ est : 0.48 Le critère de rétention est donc bien respecté : $100 \mu \mathrm{m}<3840 \mu \mathrm{m}$. Le géotextile satisfait au piégeage des sédiments. Le colmatage du géotextile permettra d'éviter le départ des matériaux inférieur à $100 \mu \mathrm{m}$.

\begin{tabular}{|c|c|}
\hline Granulométrie & Valeur en $\%$ \\
\hline Gran sup $2 \mathrm{~mm}$ & $25,92 \%$ du sédiment total \\
\hline Gran sup $500 \mu \mathrm{m}$ & $13.7 \%$ de la fraction $<2 \mathrm{~mm}$ \\
\hline Gran $500-250 \mu \mathrm{m}$ & $39.93 \%$ de la fraction $<2 \mathrm{~mm}$ \\
\hline Gran $250-163 \mu \mathrm{m}$ & $24.6 \%$ de la fraction $<2 \mathrm{~mm}$ \\
\hline Gran $163-63 \mu \mathrm{m}$ & $9.85 \%$ de la fraction $<2 \mathrm{~mm}$ \\
\hline Gran inf $63 \mu \mathrm{m}$ & $11.91 \%$ de la fraction $<2 \mathrm{~mm}$ \\
\hline
\end{tabular}

Tableau II : Répartition granulométrique du sédiment 


\begin{tabular}{|c|c|}
\hline MATIERES & TENEUR \\
\hline Mat. Calcinables & $4.11 \%$ \\
\hline C organique & $0.62 \%$ \\
\hline N total & $0.036 \%$ \\
\hline Al fusion alcaline & $53107 \mathrm{mg} / \mathrm{kg} \mathrm{sec}$ \\
\hline Fer & $31872 \mathrm{mg} / \mathrm{kg}$ \\
\hline Manganèse & $464 \mathrm{mg} / \mathrm{kg}$ \\
\hline Chrome & $62.5 \mathrm{mg} / \mathrm{kg}$ \\
\hline Zinc & $84.6 \mathrm{mg} / \mathrm{kg}$ \\
\hline Plomb & $23.5 \mathrm{mg} / \mathrm{kg}$ \\
\hline Cuivre & $35 \mathrm{mg} / \mathrm{kg}$ \\
\hline Cadmium & $3.5 \mathrm{mg} / \mathrm{kg}$ \\
\hline Nickel & $34.5 \mathrm{mg} / \mathrm{kg}$ \\
\hline Mercure & $0.05 \mathrm{mg} / \mathrm{kg}$ \\
\hline P total & $395 \mathrm{mg} / \mathrm{kg}$ \\
\hline Arsenic & $1 \mathrm{mg} / \mathrm{kg}$ \\
\hline PCB & $11.5 \mu \mathrm{kg} / \mathrm{sec}$ \\
\hline Hydrocarbures total IR & $38 \mathrm{mg} / \mathrm{kg}$ \\
\hline
\end{tabular}

Tableau III : résultats de l'analyse physico-chimique des sédiments Laboratoire d'Hydrologie et de Molismologie Aquatique-Faculté de Pharmacie-Marseille. 


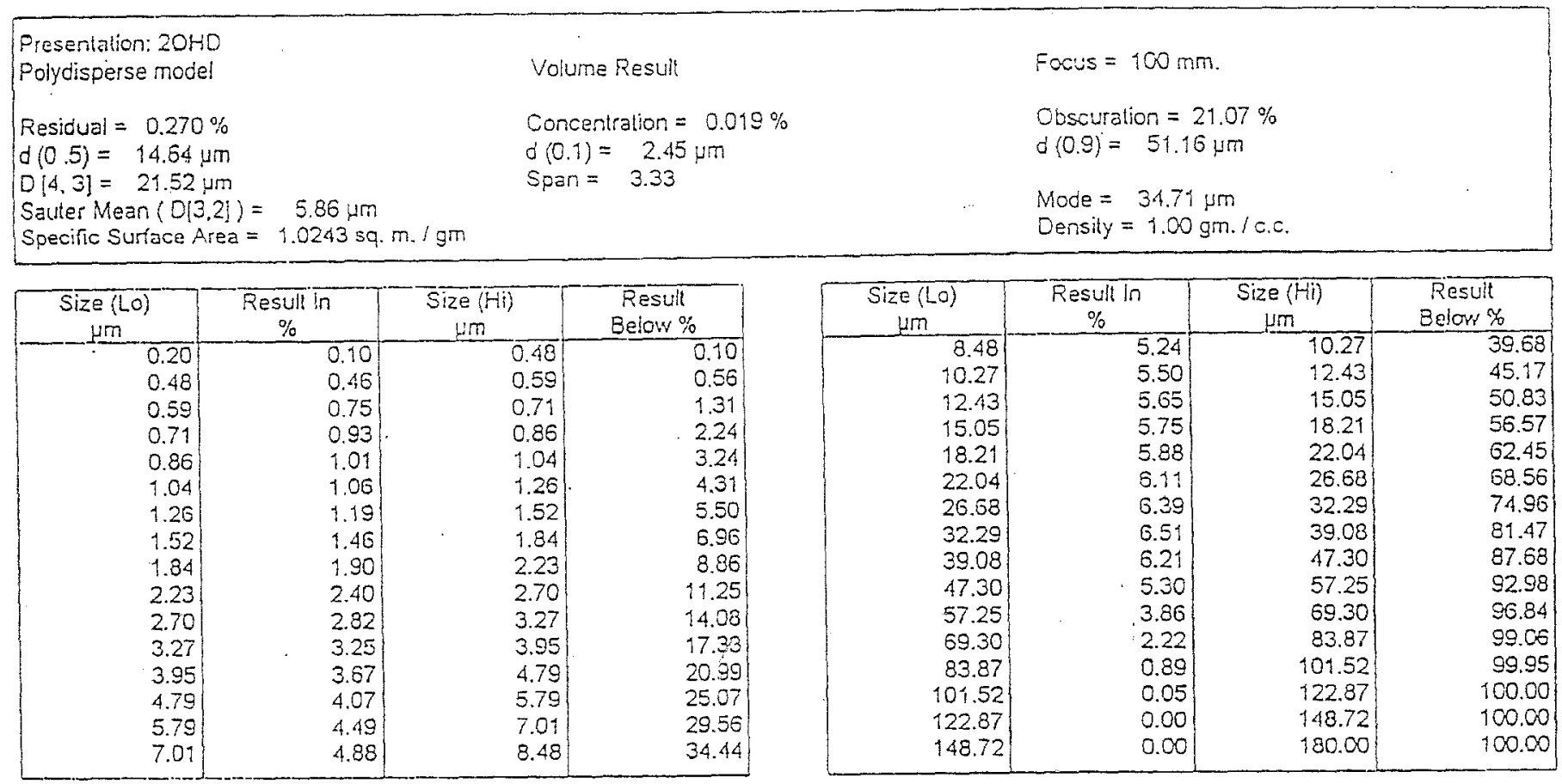

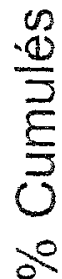

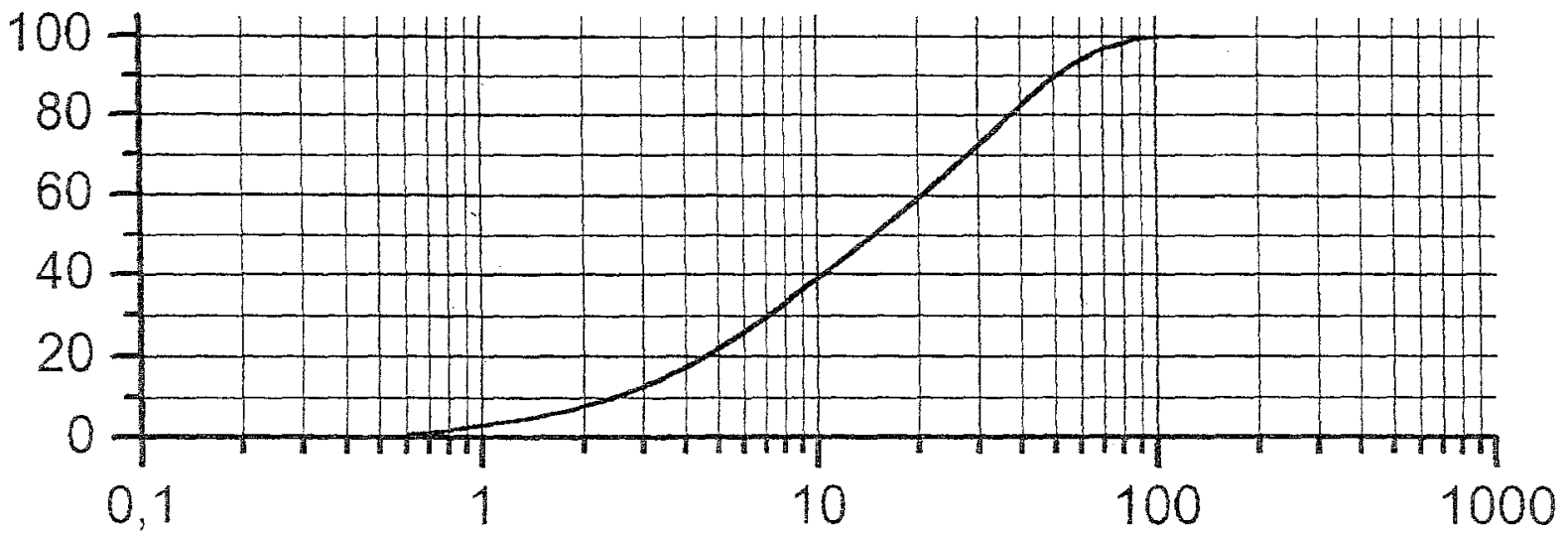

Diamètres des particules en $\mu \mathrm{m}$

Figure 1: Données et courbe granulométrique de la fraction $<100 \mu \mathrm{m}$ 


\section{$2.1 .3 \mathrm{Lesac}$}

La capacité de la soute des chalands à trappe d'immersion utilisés est en général d'environ $240 \mathrm{~m} 3$. La contenance du sac conteneur dépend de sa forme. Un sac cylindrique rond peut contenir jusqu'à $440 \mathrm{~m} 3$. Ce même sac, avec le même périmètre, en contient nettement moins quand sa section est aplatie; un sac conteneur de section elliptique ne contient plus que $200 \mathrm{~m} 3$ environ .II est apparu que la quantité maximale de sable à enfermer dans un sac conteneur qui est encore suffisamment souple pour pouvoir échapper par la trappe du chaland est d'environ $200 \mathrm{~m} 3$ (chaland de taille classique)

\subsubsection{Aménagement du Géocontainer (B)}

L'enveloppe comporte des aménagements particuliers (évents, coutures fusibles) indispensables pour assurer une immersion correcte du conteneur sans rupture du géotextile lors des deux moments critiques de la mise en oeuvre : l'immersion (chocs, évacuation de l'air) et l'arrivée au fond ( chocs, pression exercée sur le géotextile et les coutures par les matériaux).

Pour paliier aux problèmes de dégradation, les sacs conteneurs sont aménagés comme suit :

-toutes les coutures, tant les coutures industrielles que les coutures de fermeture sont renforcées, elles sont réalisées à triple rang, le fil est en polyester.

-pour les coutures de fermeture, réalisées sur chantier à la machine portative, le travail de piquage est inversé de façon à ce qu'il se fasse de l'intérieur vers l'extérieur afin que les points de couture se recouvrent mutuellement. De cette façon, un point qụi lâche reste cependant couvert par les points suivants empêchant ainsi le "dévidement" sur une grande longueur de la couture

-les sacs conteneurs sont équipés d'évents en géotextile fonctionnant comme "soupape d'échappement" pour évacuer l'air refoulé lors de l'immersion, au cours de la descente et de l'arrivée sur le fond. Ces évents en géotextile ont un diamètre de $75 \mathrm{~cm}$ et sont confectionnés avec de la toile géotextile $O_{90}$ de $1000 \mu \mathrm{m}$. Ces évents sont fixés sur la face interne du sac conteneur au moyen d'une triple couture en fil polyester. 
-Les bords des découpes faites dans le sac conteneur au droit des évents grillagés sont scellés thermiquement pour éviter qu'ils ne s'effllochent. Les évents grillagés sont disposés aux endroits ou les coutures sont le plus sollicitées

* deux évents à chaque extrémité du sac, soit deux coutures près des abouts du sac.

* trois évents sur le toit du sac

D'autres coutures servent à former un soufflet replié vers l'intérieur du sac. Ce soufflet cousu avec une couture moins résistant, en se rompant, absorbe ainsi une partie du choc.

\section{$2.2 \mathrm{La}$ barge à clapets}

La barge a un volume disponible de puits de $390 \mathrm{~m} 3$, son tirant d'eau à vide est de $0.74 \mathrm{~m}$.

\subsection{Mise en oeuvre et immersion.}

La mise en oeuvre détaillée consiste à équiper le puits de l'hydroclap en position fermée de deux nappes de géotextile qui tapisseront toute la cale du chaland et seront fixées sur les bords du clapet. Ces nappes ont pour fonction d'éviter les déchirures du sac Géocontainer (B) lorsqu'il glissera sur les parois du clapet au cours de l'immersion.(Photo 1).

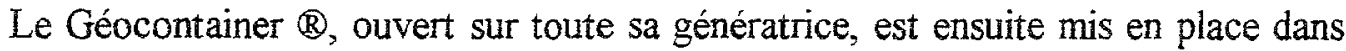
le clapet et fixé momentanément aux bords supérieurs du clapet (Photo 1). Dès lors l'opération de remplissage peut s'effectuer en réalisant une forme régulière du chargement pour répartir uniformément le poids des matériaux, ce qui permet une immersion régulière. Les $200 \mathrm{~m} 3$ sont disposés en forme de dos arrondi avec un remplissage convenable des extrémités. On dispose dans le sac des morceaux de bois et de polystyrène qui serviront à contrôler si des dégradations du géotextile intervenaient au cours de limmersion. La barge prend la mer pour se rendre sur le point de largage et durant le trajet une équipe réalise la fermeture du sac par des coutures triples, à l'aide d'une machine à coudre pontative. Le point d'immersion a été repéré grâce au G.P.S et balisé au moyen de bouées. Arrivé sur le point, les clapets sont ouverts et le sac s' immerge régulièrement. L'immersion provoque de forts remous et le dégazage au travers des évents engendre des chapelets de bulles. 


\section{Conclusion}

L'opération de conteneurisation s'est parfaitement déroulée et limmersion a montré que l'on pouvait éviter la dispersion importante des matériaux en surface lors du largage et réduire ainsi les risques de retour à la côte. Un léger panache s'est formé par la mise en suspension des matériaux qui avaient "sali" l'extérieur du

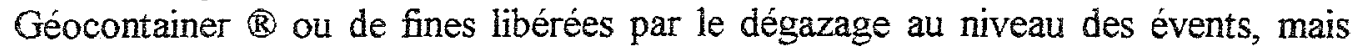
nous n'avons constaté aucune apparition des témoins de déchirure (plaquettes de bois et de polystyrène). Il nous a été impossible de vérifier le comportement du sac à son arrivée sur le fond, à la profondeur de $-180 \mathrm{~m}$. Le calcul de la vitesse, en estimant sa traînée à, de chute du sac indique une vitesse limite d'environ $4.2 \mathrm{~m} / \mathrm{s}$ soit $15 \mathrm{~km} / \mathrm{h}$. Il semble probable qu' elle n'ait pas entraîné de dégradation importante. Nous n'avons constaté aucune apparition des témoins de déchirure mais ils peuvent avoir dérivé loin du point d'impact entre deux eaux pour émerger hors de notre vue. Cette première expérience est très encourageante mais les prochaines expériences françaises devront permettre de vérifier en détail le

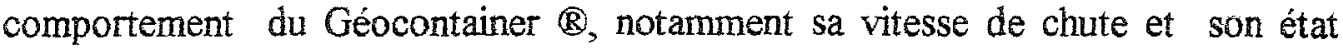
après limpact au fond. La vitesse de chute pourra être vérifiée par le chronométrage du passage du Géocontainer ß à plusieurs profondeurs grâce aux plongeurs ou par le calcul après avoir déterminé précisement le coefficient de trainée. Il sera nécessaire, si la profondeur d'immersion est aussi importante, d'utiliser un R.O.V. pour filmer les Géocontainers (B) sur le fond. D'autre part, il sera nécessaire de prévoir des grillages d'évents dont la porométrie permettent le dégazage en assurant le confinement de tous les matériaux et particulièrement des fines auxquelles sont attachés les risques importants de pollution. Des essais en modèles réduits seront difficilement réalisables et seuls les essais grandeur nature pourront apporter les informations souhaitées. Nous restons donc confrontés aux difficultés de réalisation d'essais grandeur nature, qui sont dans un premier temps d'ordre financier.

Référence bibliographique :

[1] JAGT H. J. - Protection. au moyen de Géocontainers $\$$, de la berge du bras de la Meuse dénommmé "OUDE MAAS" - Rijkswaterstaat, Servive des cours d'eau et des voies navigabies des Pays -Bas - 1988, p.21 et Annexes 1 à 11 


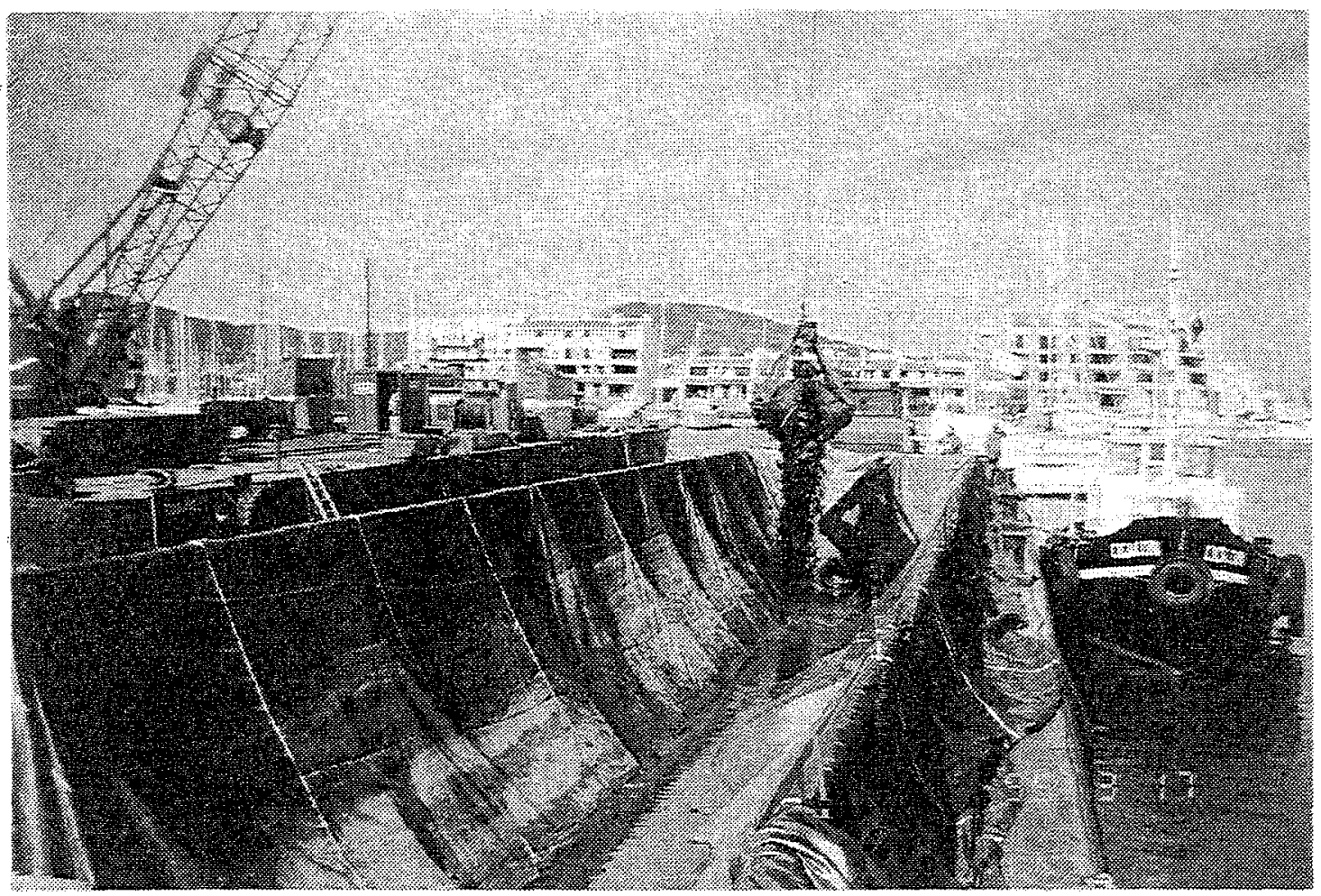

Photo 1: Le Géocontainer $B$ est mis en place, ouvert sur toute sa génératrice, puis fixé à l'aide de serre-joints sur le plat-bord du puits. Le remplissage peut alors débuter en réalisant une forme de dos arrondi. 